\title{
IP and Broadcasting Systems Convergence
}

\author{
Georgios Gardikis, ${ }^{1,2}$ George Xilouris, ${ }^{2}$ Marie-Jose Montpetit, ${ }^{3}$ \\ Alessandro Vanelli-Coralli, ${ }^{4}$ and Daniel Negru ${ }^{5}$ \\ ${ }^{1}$ Institute of Informatics and Telecommunications, NCSR "Demokritos", Agia Paraskevi 15310, Greece \\ ${ }^{2}$ Technological Educational Institute of Crete, Heraklion 71500, Greece \\ ${ }^{3}$ Research Laboratory of Electronics, MIT, Cambrige, MA 02139-4307, USA \\ ${ }^{4}$ ARCES, University of Bologna, 40136 Bologna, Italy \\ ${ }^{5}$ LaBRI-CNRS, University of Bordeaux, 33405 Talence Cedex, France
}

Correspondence should be addressed to Georgios Gardikis, gardikis@iit.demokritos.gr

Received 31 December 2010; Accepted 31 December 2010

Copyright (c) 2010 Georgios Gardikis et al. This is an open access article distributed under the Creative Commons Attribution License, which permits unrestricted use, distribution, and reproduction in any medium, provided the original work is properly cited.

The vision of the Internet of the Future for service ubiquity dictates that end users should be able to access servicesmostly media-oriented ones-at any time from a variety of networks, including mobile, broadband, and broadcast. In order to achieve network interworking and service ubiquity, IP arises as a key element for the realisation of a unified/fusion environment which enables the convergence/synergy between traditional and emerging technologies. In this context, IP can be also seen as the "gluing factor" in the rapidly progressing convergence between the technologically different sectors of Networking and Broadcasting.

This convergence, witnessed both at technological and service levels, is mainly empowered by the evolution of broadcasting standards (DVB, ISDB, ATSC, CMMB) and the recent advances in IP networking.

In this way, a broadcasting platform is no longer restricted to transmitting "bouquets" of TV programs. The ability to include IP services into the broadcast multiplex, along with the large coverage area and the high bit rate capabilities, allows broadcasting systems to constitute flexible broadband IP networking infrastructures, complementing existing and emerging wireless access networks such as $3 \mathrm{G}$, WiMAX, and LTE.

Conversely, the provision of broadcast TV services over IP networks and over the Internet is further fading the borders between the IP/Networking and Broadcast worlds.

The aim of this special issue has been to include recent research efforts focusing on the convergence between the IP and Broadcasting systems, that is, the provision of IP services over broadcasting platforms and vice versa, the streaming of $\mathrm{A} / \mathrm{V}$ broadcasting services over IP networks.

re-strict-edAn interesting aspect is the use of broadcasting platforms for regional-area IP networking, focusing on rural/underdeveloped areas. In this context, the paper entitled "Hybrid terrestrial-satellite DVB/IP infrastructure in overlay constellations for triple-play services access in rural areas" presents the concept, implementation, and experimental evaluation of a novel, dual-layer architecture, where rural customers are served by an interactive DVB-T platform. As an overlay, DVB-T service networks are interconnected by a satellite DVB-S2/DVB-RCS network, which acts as backhaul.

Video traffic is dominant across convergent IP/broadcast networks, and its proper transport and presentation is critical for end user satisfaction. The paper with the title "Video quality prediction models based on video content dynamics for H.264 video over UMTS networks" introduces an emulated loss model for H.264 video over data networks, focusing on 3G, and proposes an Adaptive Neural Fuzzy Inference System (ANFIS) and also a second model based on nonlinear regression analysis in order to predict the quality of the video, as perceived by the viewer.

DVB-T2 is the new cutting-edge technology in digital media terrestrial broadcasting. Its Physical Layer Pipes (PLP) feature enables the transmission of different streams with different physical-layer characteristics. The paper entitled "Cross-layer optimization of DVB-T2 system for mobile services" combines the PLP feature with Scalable Video Coding 
in order to achieve cross-layer optimisation, with a variety of options, and maximise the efficiency of video delivery over DVB-T2 to diverse reception environments.

Delivery of IPTV streams in multicast environments is examined in "Design of an IPTV multicast system for internet backbone networks." The authors present the design of an IPTV multicast system for the Internet backbone network and study it through extensive simulations. Traffic scheduling, shaping, and regeneration techniques are employed at the source and the destination nodes to achieve optimal delivery and a near-perfect end-to-end QoS.

The issue of transporting IP data over a DVB platform is investigated in "A framework for an IP-based DVB transmission network". The paper studies the GS (Generic Stream) and GSE (Generic Stream Encapsulation) schemes which allow direct transport of IP traffic, eliminating the use of the legacy MPEG-2 Transport Stream. Novel approaches are introduced, which replace MPEG-2 signalling with GSEcompliant methods and allow the DVB network to be seamlessly included in an all-IP architecture and act as part of the Internet.

Network-level Quality of Service (QoS) is a critical factor for user satisfaction in IPTV systems. In the paper titled "On the QoS of IPTV and its effects on home networks" the authors propose a queuing model for IPTV systems and use it to analyze the impact of IPTV traffic on other home-network applications. Results show that standard TCP congestion control is not adequate for this purpose and a tuning/optimisation procedure is proposed.

In the same context (i.e., QoS assurance in media delivery), the paper with the title "Performance evaluation of triple play services delivery with E2E QoS provisioning" presents an enhanced core and access network architecture, featuring a WiMAX access network and allowing end-to-end QoS provision. Traffic-class mapping in the core network, following a DiffServ/MPLS approach, is extended to the access part, using native WiMAX QoS support. The efficiency of the mechanism is validated in a real demonstrator environment, where media streams are prioritised over background traffic and properly delivered.

Satellite broadcast networks can be remarkably efficient when used for data backhaul connectivity. The paper entitled "Network performance evaluation of abis interface over DVB$S 2$ in the GSM over satellite network" investigates the use of a satellite broadcast DVB-S2 channel for interconnecting a GSM Base Station Controller (BSC) with a remote Base Transceiver Station (BTS), that is, for implementing the Abis interface. Extensive ns2-based simulations are employed to optimise transmission parameters and to determine satellite bandwidth and cost requirements for different network setups.

\section{Acknowledgments}

We would like to thank all the authors who submitted their work to this special issue and the reviewers for their timely and valuable assistance. We are also grateful to the editing and support personnel of the Journal who helped make this special issue a reality and especially to the Editor-in-Chief, Dr. Luo, for his invitation and his support throughout the entire process.

Georgios Gardikis

George Xilouris

Marie-Jose Montpetit

Alessandro Vanelli-Coralli

Daniel Negru 

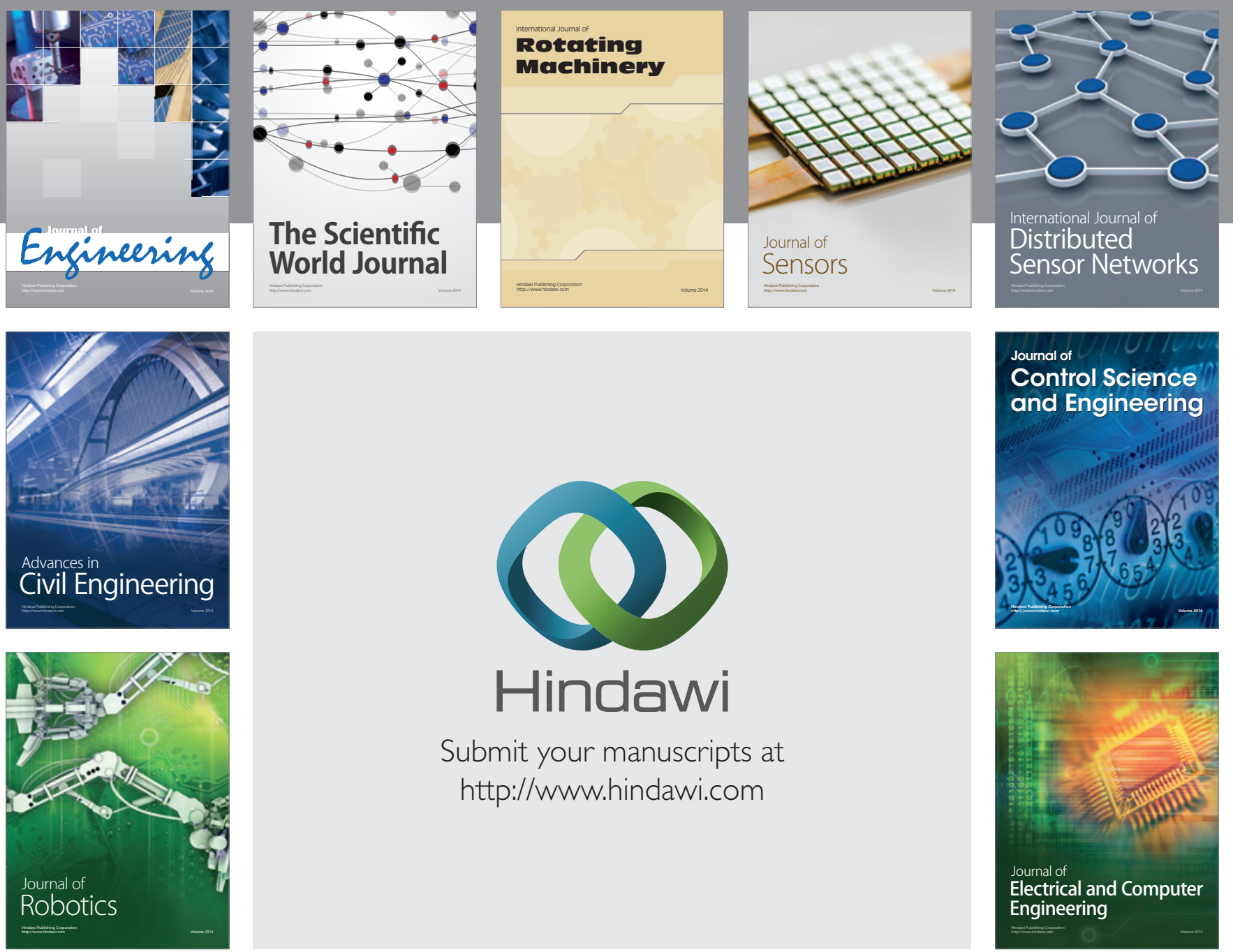

Submit your manuscripts at

http://www.hindawi.com
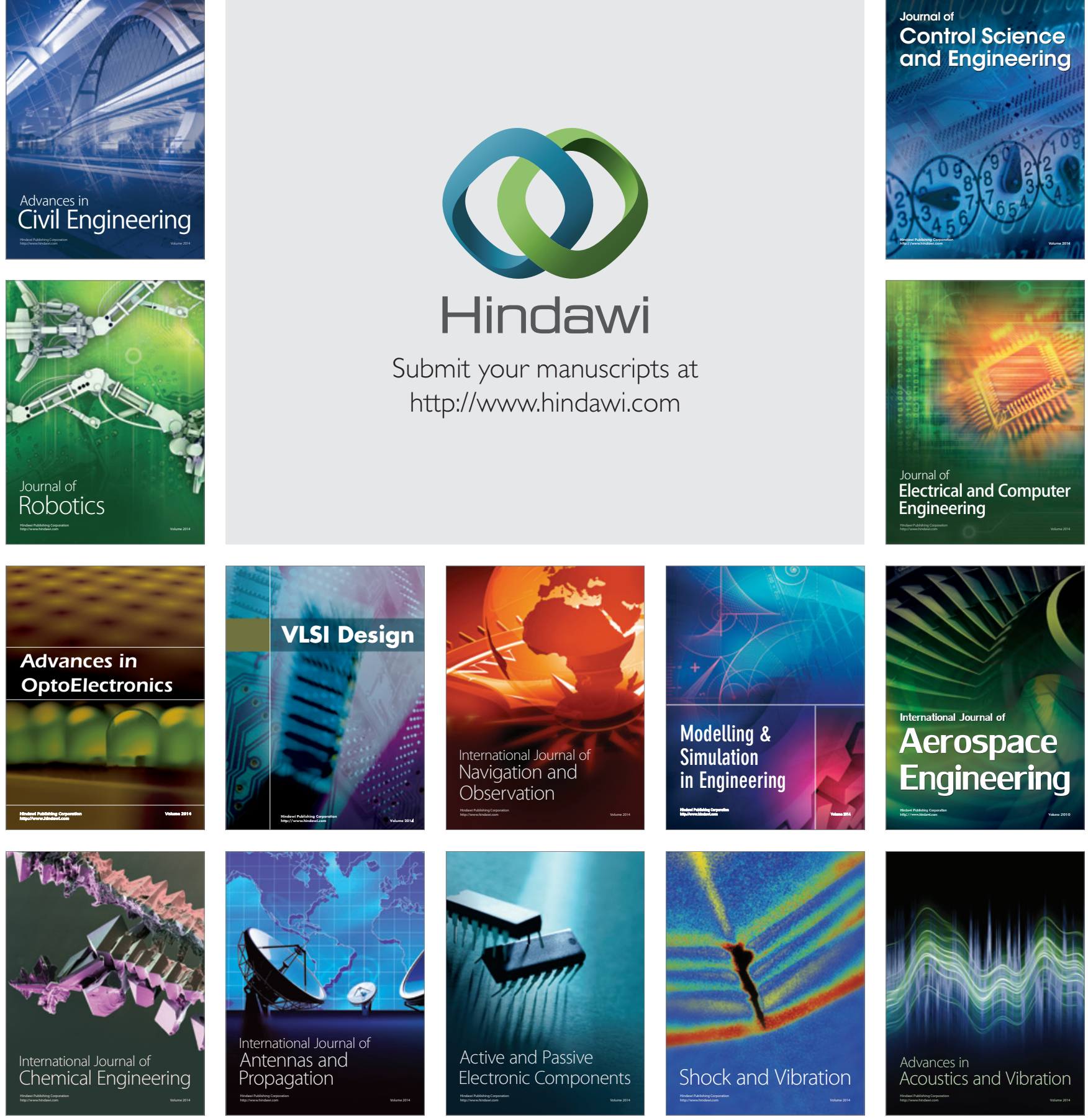